\title{
Motor switching abilities in Parkinson's disease and old age: temporal aspects
}

\author{
Meir Plotnik, Tamar Flash, Rivka Inzelberg, Edna Schechtman, Amos D Korczyn
}

Sreratzki Chair of Neurology, Tel-Aviv University, Tel-Aviv, Israel

M Plotnik

A D Korczyn

Department of Applied Mathematics and Computer Science, the Weizmann Institute of Science, Rehovot, Israel

M Plotnik

T Flash

R Inzelberg

Department of Neurology, Tel-Aviv Sourasky Medical Center, Tel-Aviv, Israel R Inzelberg

A D Korczyn

Computer Center, the Weizmann Institute of Science, Rehovot,

Israel

E Schechtman

Correspondence to: Dr Amos D Korczyn, Department of Neurology, Sackler Faculty of Medicine, Tel-Aviv University, Ramat-Aviv, 69978, Israel.

Received 20 December 1996 and in revised form 26 November 1997

Accepted 16 February 1998

\begin{abstract}
Objectives - To investigate capabilities of arm trajectory modification in patients with Parkinson's disease and elderly subjects using a double step target displacement paradigm.

Methods-Nine patients with Parkinson's disease and seven age matched control subjects were instructed to move a stylus towards visual targets presented on a digitising table. Within each session, in some trials the target location was changed before initiation of movement and the subjects were to modify their movements towards the new target (switching trials). In other trials the target location was not changed (control trials). This procedure was repeated for four different target configurations, using interstimulus time intervals of six different durations. The subjects' hand trajectories were recorded and their kinematic characteristics were analysed.
\end{abstract}

Results-In switching trials, about $40 \%$ of the movements were aimed directly toward the final target location in both groups. When the trajectories were initially directed toward the first target and then modified toward the second, the reaction time (RT) to the second stimulus $\left(\mathrm{RT}_{2}\right)$ was longer than to the first stimulus $\left(\mathrm{RT}_{1}\right)$. The $\mathrm{RT} \mathrm{T}_{2} / \mathrm{RT}_{1}$ ratio was significantly larger in patients with Parkinson's disease than in healthy elderly subjects.

Conclusions-Patients with Parkinson's disease and elderly subjects are substantially slower in responding to a required modification of their movement than in responding to the required movement initiation. Patients with Parkinson's disease have impaired capabilities in processing simultaneously the motor responses to two visual stimuli presented in rapid succession.

(F Neurol Neurosurg Psychiatry 1998;65:328-337)

Keywords: Parkinson's disease; motor switching; basal ganglia; kinematics

Patients with Parkinson's disease are known to have difficulties in planning and performing sequential or simultaneous motor acts. In studies in which sequential motor acts were investigated (for example, tapping a keyboard), patients with Parkinson's disease were impaired in their ability to preprogramme the complete sequence. ${ }^{12}$ The shift from one segment of the sequence to the next was also more difficult for patients with Parkinson's disease. ${ }^{3}$ In studies in which simultaneous motor acts were required, patients with Parkinson's disease were slower and less accurate in their performance. ${ }^{45}$

The term "complex movements" is used by different investigators to refer to various motor capabilities. The performance of simultaneous motor acts, sequences of movements, or the modification of ongoing movements, may reflect different aspects of basal ganglia function. In the present study we investigated the performance of complex motor tasks by studying both simple reaching movements and the modification of ongoing arm movements.

In recent years the modification of arm trajectories in response to sudden displacements of target location was studied by several research groups. ${ }^{6-11}$ Originally it was suggested that motor responses are prepared one at a time - that is, by a single channel of stimulusresponse processing. Hence, while the brain is preparing an appropriate motor response to the first stimulus, the second must wait its turn. The time delay before dealing with the second stimulus while its antecedent is still being processed is termed the psychological refractory period (PRP; for a review see Poulton ${ }^{12}$ ).

Nevertheless, it was shown that the single channel model does not always hold, as is the case, for example, of the double-step target displacement paradigm. Several studies disclosed that when subjects were required to move their hand towards a visual target the location of which was unexpectedly changed, the reaction times (RTs) to the second stimulus $\left(\mathrm{RT}_{2}\right)$ were only slightly prolonged compared with the RTs to the first stimulus $\left(\mathrm{RT}_{1}\right)$. This finding was made both in primates ${ }^{6}$ and in humans, ${ }^{911}{ }^{13-15}$ giving rise to the hypothesis that the processes subserving the preparation of the motor response to the second stimulus are carried out in parallel with those involved in the preparation or execution of the response to the first.

Several alternative models have been suggested for the mechanisms subserving arm trajectory modification. According to a model proposed by Flash and Henis, ${ }^{9}$ the modification of planar arm movements involves parallel planning and superposition of two trajectory plans. One motor plan specifies the hand trajectory from its initial position to the first target position, and the other specifies the trajectory between the first and second target positions. These two programmes are independently planned, and are vectorially added starting at the point in time when the second motor plan is ready. This summation of two separate motor plans results in smoothly modi- 
Table 1 Description of parkinsonian patients (PD) and age matched control group (CS)

\begin{tabular}{|c|c|c|c|c|c|c|c|}
\hline Subject & Sex & Age & $\begin{array}{l}\text { Hoehn and Yahr } \\
\text { scale }\end{array}$ & $\begin{array}{l}\text { Disease } \\
\text { duration (y) }\end{array}$ & $\begin{array}{l}\text { Predominant } \\
\text { symptom }\end{array}$ & Medication & $\begin{array}{l}\text { Hours off } \\
\text { drugs }\end{array}$ \\
\hline \multicolumn{8}{|l|}{ PD: } \\
\hline PD1 & $M$ & 56 & II & 18 & $\mathrm{~T}, \mathrm{~B}$ & $\mathrm{D}, \mathrm{O}, \mathrm{A}$ & 16 \\
\hline PD2 & $\mathrm{F}$ & 75 & III & 4 & $\mathrm{R}, \mathrm{B}$ & $\mathrm{Br}, \mathrm{D}, \mathrm{S}$ & 16 \\
\hline PD3 & M & 78 & II & 1 & $\mathrm{~T}, \mathrm{R}$ & $S, \operatorname{Tr}$ & 15 \\
\hline PD4 & M & 78 & III & 7 & $\mathrm{R}, \mathrm{T}, \mathrm{B}$ & $\mathrm{D}, \mathrm{Br}, \mathrm{Tr}$ & 15 \\
\hline PD5 & M & 82 & II & 7 & $\mathrm{R}$ & $\mathrm{D}, \mathrm{Br}, \mathrm{S}$ & 21 \\
\hline PD6 & $\mathrm{F}$ & 77 & III & 9 & $\mathrm{~T}, \mathrm{~B}$ & $\mathrm{D}, \mathrm{A}, \mathrm{Tr}, \mathrm{S}$ & 4.5 \\
\hline PD7 & M & 77 & III & 3 & $\mathrm{R}, \mathrm{T}, \mathrm{B}$ & $\mathrm{D}, \mathrm{Br}$ & 15 \\
\hline PD8 & $\mathrm{F}$ & 66 & II & 5 & $R, B$ & $\mathrm{D}, \mathrm{Br}, \mathrm{A}$ & 17 \\
\hline PD9 & $M$ & 79 & II & 4 & $\mathrm{~T}, \mathrm{R}$ & $\mathrm{D}$ & 36 \\
\hline \multicolumn{8}{|c|}{ Controls: } \\
\hline CS1 & M & 59 & - & - & - & - & - \\
\hline $\mathrm{CS} 2$ & M & 72 & - & - & - & - & - \\
\hline CS3 & $\mathrm{F}$ & 77 & - & - & - & - & - \\
\hline CS4 & $\mathrm{F}$ & 67 & - & - & - & - & - \\
\hline CS5 & $\mathrm{F}$ & 78 & - & - & - & - & - \\
\hline CS6 & M & 83 & - & - & - & - & - \\
\hline CS7 & M & 83 & - & - & - & - & - \\
\hline
\end{tabular}

$\mathrm{B}=$ bradykinesia; $\mathrm{R}=$ rigidity; $\mathrm{T}=$ tremor; $\mathrm{A}=$ amantadine; $\mathrm{Br}=$ bromocriptine; $\mathrm{D}=$ levodopa/carbidopa; $\mathrm{O}=$ orphenadine; $\mathrm{S}=$ selegiline; $\operatorname{Tr}=$ trihexyphenidyl.

fied hand trajectories. Alternative models for modification of arm trajectory also assume parallel processing of the motor responses to the two stimuli. ${ }^{76}$ This assumption is based on the idea that complicated motor actions emerge as a result of the simultaneous preparation of different movement elements, which are assembled together before execution of movement. This notion is supported by the parallel architecture of the cortical-basal ganglia motor circuitry, and the massive neural connectivity within the elements of these circuits. ${ }^{17} 18$

\section{Materials and methods}

SUBJECTS

Nine patients with Parkinson's disease (six men) at stages II and III on the Hoehn and Yahr scale, ${ }^{19}$ and seven healthy subjects (four men) participated in this study. The mean age of the patients was 74.2 (SD 8.1), range 56-82, and the mean age of the control subjects was 74.1 (SD 8.8), range 59-83. All subjects were right handed. The patients with Parkinson's disease were off medication for several hours (mean 17 (SD 8.2), range 4.5-36). Data about the patients and control subjects are presented in table 1.

\section{EXPERIMENTAL APPARATUS}

Figure $1 \mathrm{~A}$ (left) illustrates the experimental apparatus, which consisted of a $50.8 \times 50.8 \mathrm{~cm}$ digitising table. Underneath the transparent surface of this table, light emitting diodes were installed and were used as visual targets. The sequence of target illumination was controlled by a computer program which determined the specific light emitting diode to be illuminated and the duration of its illumination. A pen-like stylus was held by the subject, and enabled the localisation of the hand coordinates $x, y$ with an accuracy of $0.25 \mathrm{~mm}$ at a rate of $100 \mathrm{~Hz}$. The path of the stylus was stored in a computer, and the hand trajectories were graphically reconstructed off line, using a SUN 3/50 work station. A Lagrange polynomial differentiation was used to obtain the $x$ and $y$ components of the hand velocity.
EXPERIMENTAL PARADIGM

The present study followed a study of arm trajectory modification in young adults, performed previously in our laboratory ${ }^{9}$ using an identical paradigm, and care was taken to provide the subjects with identical instructions. Each subject was seated in front of the table, holding the stylus in his or her right hand. The subject's shoulder was restrained to the back of the chair by straps, and the wrist was braced to eliminate movements of the wrist joint. Thus movements were confined to elbow and shoulder rotations in the horizontal plane at the level of the subject's shoulder. Visual feedback from the moving limb was avoided by performing the experiment in complete darkness. ${ }^{9}{ }^{20}$

Each experiment consisted of several sessions, each composed of 26 movement trials. Figure 1A (right side) shows the spatial arrangement of the light emitting diode targets that were used in our experiments. At the beginning of each trial, the hand was located at the initial position $\mathrm{O}$. At time $\mathrm{t}=0$ this light emitting diode was switched off and a new target at one of two equally probable locations, A or B, was illuminated. This target either remained lit (a control trial with a probability 0.38 ) or was shifted (a switching trial) to one of two equally probable positions as follows: When position A was first illuminated, the target was switched either to $\mathrm{B}$ or $\mathrm{C}$ as final targets. When position B was first illuminated, the target was switched either to position $\mathrm{C}$ or D. Positions A or B (during the switching trials) will be referred to as first targets and final positions $\mathrm{B}, \mathrm{C}$, or D in these trials as final targets. Taken together, each experimental session consisted of two types of control trials: OA or OB (10 altogether in each session), and of four types of switching trials: $\mathrm{OAB}, \mathrm{OAC}, \mathrm{OBC}$, or OBD (16 altogether in each session). Six different interstimulus intervals (ISIs) were used in the experiments $(50,100,150,250$, 300 , or $350 \mathrm{~ms}$ ). As part of the analysis (see below), data concerning direct hand trajectories between the initial hand position and the targets $\mathrm{C}$ and $\mathrm{D}$ were required. Therefore at the end of the sessions involving switching tri- 


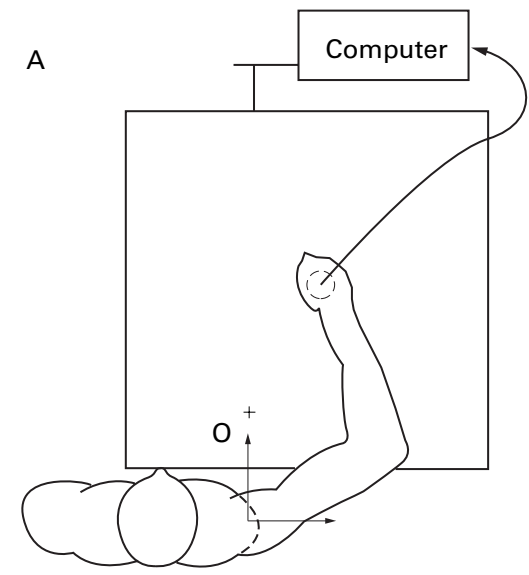

B

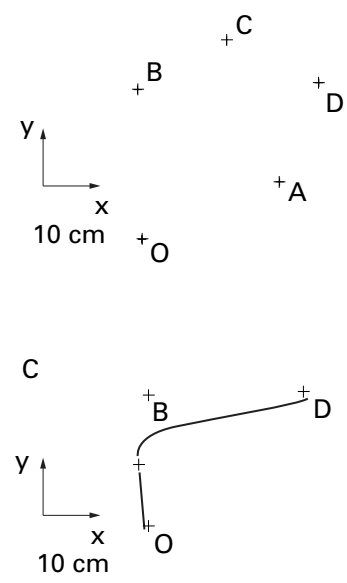

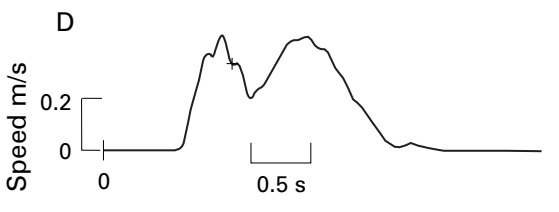
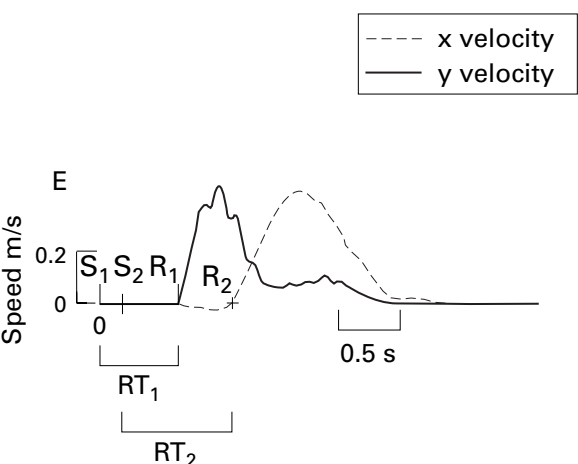

Figure 1 Experimental apparatus and kinematic analysis. (A) Schematic drawing of the seated subject in front of the apparatus. The initial position is marked by $O$. (B) The five target locations that were used in the experiments. The control tasks were composed of configurations $O A$ and OB. Switching tasks were composed of configurations $O A B, O A C, O B C$, and $O B D$. (C) Double segment movement OBD. (D) The path; tangential velocity. The cross denotes the point at which the subject responded to the appearance of the target $D$. (E) Axial velocitiy profiles as a function of time. At $t=0$ target $B$ was lit $\left(S_{\nu}\right)$ and after $150 \mathrm{~ms}$ target $D$ was lit $\left(S_{2}\right)$. At the time point $R_{1}$, the hand started moving in the direction of target $B(y$ velocity), and the interval $S 1 R 1$ denotes the first reaction time, $R T_{1}$. At $R_{2}$, the $x$ component of the velocity increases above zero. This point is marked as the point in time when the subject responded to the appearance of the second target. Thus $R T_{2}$ corresponds to the interval $S_{2}-R_{2}$. The interstimulus interval (ISI) $=S_{2}-S_{1}$.

als, for each subject, movement trials involving either OC or OD target configurations (15 of each) were recorded.

The instructions to the subject were to hold the stylus at the initial position $\mathrm{O}$. Then, on the turning off of the lit target and the simultaneous illumination of the new target (A or B), to move the stylus toward the second. On further change in target location (in switching trials) the subject was to move his or her hand toward the newly presented target location. To mimic as much as possible a spontaneous movement, no instructions concerning movement speed, accuracy, or the type of trajectory to be generated were given. Before starting the recording sessions, the subjects performed a practice session in which we verified that the task was well understood.

\section{DATA ANALYSIS}

\section{Qualitative assessment}

Movement trials that violated the instructions given to the subjects were excluded from the analysis. These included, for example, trials in which the subjects raised the stylus from the surface of the table. In the switching trials, the relatively few cases $(1.7 \%)$ in which the RT to the appearance of the first target was shorter than the ISI were also omitted from the analysis, so as to deal only with target shifts occurring within the preparation time.

Performance classification - The hand trajectories during control trials were categorised as (1) regular movements, and (2) errors (responses such as moving toward a non-existent target). The switching trials were categorised as (a) double segment movements. These movements consisted of the following segments. The first was directed either toward the first target or in between the first and final targets. The second segment started when the modification of movement direction toward the final target location was detected. This point in time could be clearly identified by detecting a veer in the direction of the hand velocity vector (defined as $\theta=\arctan v_{y}$ over $v_{x}$, where $v_{y}$ and $v_{x}$ are the $y$ and $x$ components of the hand velocity (vector). These two segments were represented by a bimodal tangential velocity profile. Some of the double segment movements contained a pause (defined to be at least $20 \mathrm{~ms}$ of duration) before the direction of the trajectory was modified toward the final target, whereas others were continuously modified. (b) Direct movements towards the final target. These movements consisted of a single segment and were directed toward the final target location. (A few cases in which the hand initially moved to an intermediate direction in between the two targets, and then gradually curved toward the final target were categorised as direct movements as no specific modification in the direction of the hand velocity vector could be detected.) (c) Errors-that is, movements that were performed in a way that deviated from the expected for the specific trial type.

\section{Quantitative assessment}

Temporal characteristics-The RTs for each movement were determined as the time interval between the illumination of the target and movement initiation. The criteria applied to determine the values of $\mathrm{RT}_{1}$ and $\mathrm{RT}_{2}$ in double segment movements are explained in figure $1 . \mathrm{RT}_{1}$ was defined in the same manner as RT in control tasks - that is, the time interval between the appearance of the first stimulus $\left(\mathrm{S}_{1}\right)$, and the initiation of the first response $\left(R_{1}\right)$. The second reaction time, $R_{2}$, was defined as the time interval elapsing between the appearance of the second stimulus $\left(\mathrm{S}_{2}\right)$ and the point in time when the direction of the 
velocity vector turned toward the final target minus the ISI that was used in that trial (the time interval $\mathrm{S}_{2}-\mathrm{R}_{2}$ ).

The ratio $\mathrm{RT}_{2} / \mathrm{RT}_{1}$ was calculated for each double segment movement. Hence we could assess the prolongation of the response to the second stimulus normalised by the RT to the first stimulus. The use of this normalisation overcomes intersubject RT variability, disclosed especially in elderly subjects, as well as the influence of factors that may vary during the experimental session (for example, alertness). Movement time (MT) was defined as the time interval elapsing between movement initiation $\left(\mathrm{RT}_{1}\right.$, and $\mathrm{RT}$ for double segment movements and control and direct movements respectively) and the time when the tangential velocity again reduced to zero. The time interval between the change in target location and the initiation of the movement is termed modification time (denoted as D).

Psychological refractory period (PRP) calculation-According to the single channel theory, the PRP is quantitatively reflected by a prolonged RT to the second stimulus. This prolongation can be expressed as follows:

$$
\mathrm{RT}_{2} \exp =\mathrm{RT}_{1}{ }^{\prime}+\mathrm{RT}_{2}{ }^{\prime}-\mathrm{ISI}
$$

where $\mathrm{RT}_{2} \exp$ denotes the expected value of $\mathrm{RT}_{2}$ according to the single channel theory, and $\mathrm{RT}_{1}{ }^{\prime}$ and $\mathrm{RT}_{2}{ }^{\prime}$ are the RTs to the first and second stimuli respectively, had each been presented alone. ${ }^{21}$ Equation (1) evaluates the refractory period: $\mathrm{RT}_{1}{ }^{\prime}-\mathrm{ISI}$; this implies the validity of this evaluation only to cases whereby $\mathrm{RT}_{1}{ }^{\prime}>\mathrm{ISI}$. Values for $\mathrm{RT}_{2} \exp$ were derived as follows: the mean values of RTs (of a particular subject) obtained in the corresponding control trial were substituted for the variables $\mathrm{RT}_{1}{ }^{\prime}$ and $\mathrm{RT}_{2}{ }^{\prime}$ (for example, in target configuration $\mathrm{OAB}$, the mean RT for the OA control movement was substituted for $\mathrm{RT}_{1}{ }^{\prime}$ and the mean RT for the OB control movements was used for $\left.\mathrm{RT}_{2}{ }^{\prime}\right)$. The ISI value of that particular trial was subtracted from this sum according to equation (1). Hence the PRP is specifically expressed by the term $\mathrm{RT}_{1}{ }^{\prime}-\mathrm{ISI}$, and has a positive value when $\mathrm{RT}_{1}{ }^{\prime}>$ ISI, which corresponds to the case investigated in the present study.

The data that were obtained in the control movements OC and OD (which were measured in separate sessions - see above), showed that the values of RTs in these configurations were comparable with the values of RTs in the other control trials.

\section{STATISTICAL ANALYSIS}

The proportions of the different movement categories (the percentage of movements that could be classified as direct movements, double segment movements, and errors) in the switching trials for each subject were analysed by univariate analysis of variance (ANOVA). The model includes four main effects: three fixed (group-patients/control, target configuration - four levels, and ISI - six levels), and one random (subject nested within the group).

The temporal variables, MTs and RTs $\left(\mathrm{RT}_{1}\right.$, $\mathrm{RT}_{2}$, and $\mathrm{RT}_{2} / \mathrm{RT}_{1}$ ), were found to be positively correlated within some of the subjects. There- fore, the temporal parameters were analysed first by multivariate analysis of variance (MANOVA) to analyse the entire vector of variables simultaneously, and then by the univariate ANOVA procedure to analyse each separately. The model for analysing the data obtained in the switching trials was similar to the one described above. In the control trials the model included three main effects, two fixed (group and target configuration), and one random (subject nested within the group). Both types of analysis (MANOVA and ANOVA) were performed using a general linear model with repeated measures. Whenever an interaction between the effects was found not to be significant $(p>0.1)$, it was removed from the ANOVA models (both for the proportions of the different movement categories and for the temporal parameters). Other statistical tests and procedures which were used are described in the results section.

\section{Results}

QUALITATIVE ASSESSMENT

Examples of various movements performed in control and switching trials are shown in fig 2 . In the switching trials of patients with Parkinson's disease $(n=1495)$, the proportion of double segment movements was on average $51 \%$ (SEM 8.3\%), which was lower than for the control subjects $(61 \%$, SEM $8 \%, n=1033)$. The proportions of direct movements were $45 \%$ (SEM 8.6\%) and 34\% (SEM 8.1\%) for the patients with Parkinson's disease and control subjects respectively, with the remainder being errors. The difference in proportions between the groups (for each category) was not significant. Both Parkinson's disease and control subjects had a relatively larger number of errors during the switching tasks compared with control trials (two way ANOVA, $p<0.02$ for patients with Parkinson's disease, who performed $1 \%$ of errors in control trials, and $\mathrm{p}<0.04$ for control subjects, who performed $1.65 \%$ errors in control trials).

\section{Double segment movements}

The two movement segments were well reflected in the double peaked tangential velocity profiles of the movement trajectories (figs 1, 2 C, D, E). Trajectory modifications were clearly seen both in the path and in the direction of the hand velocity vector of the movement. No qualitative difference in the kinematic form of double segment trajectories was found between the groups.

The proportions of paused movements (for example, fig $2 \mathrm{E}$ ) were similar for both groups (about $16 \%$ of all double segment movements). The proportion of paused double segment movements increased with the duration of the ISI $(0.001<p<0.01$, fig $3 \mathrm{~A})$. On average, the pauses of modified hand trajectories of the patients with Parkinson's disease occurred at $46 \%$ (SEM 4\%) of the distance between the initial and the first target in patients with Parkinson's disease, and at $51 \%$ (SEM $4 \%$ ) of this distance in control subjects. The difference was not significant. 
Sub : CS4

Con: OA

A

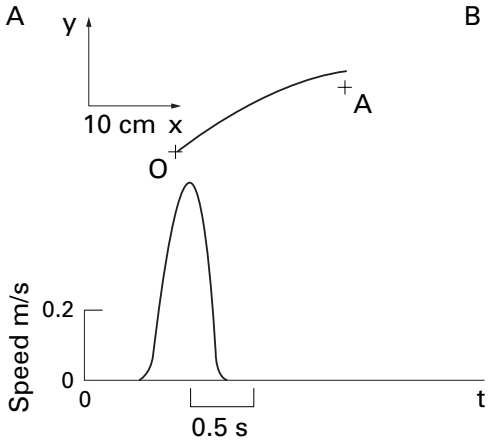

B

Con : OB
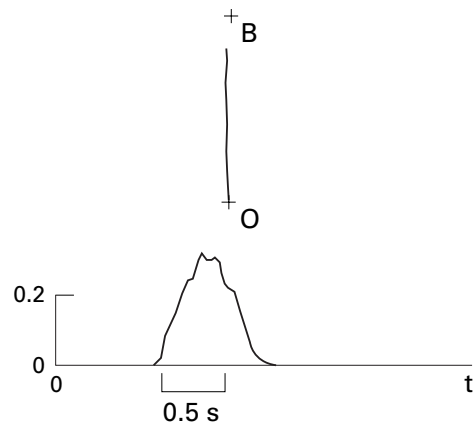
Sub : PD7
Con : $\mathrm{OAB}$
ISI : 300
D Sub : PD3
Con : OAB
ISI : 100
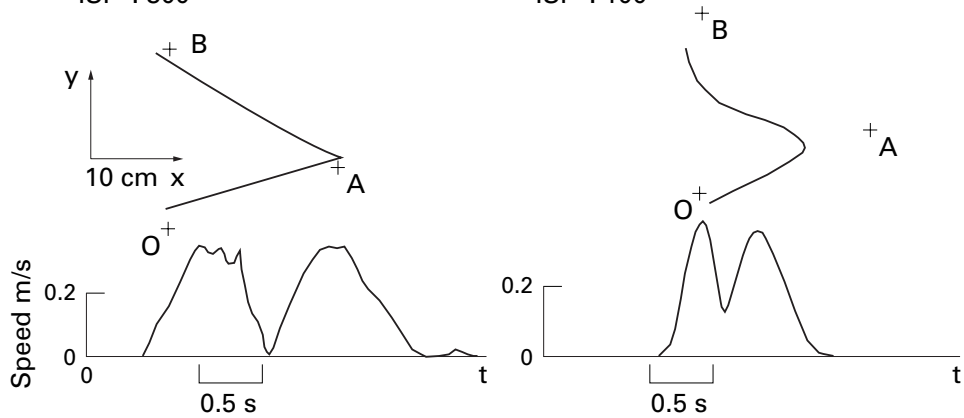

E Sub : CS5

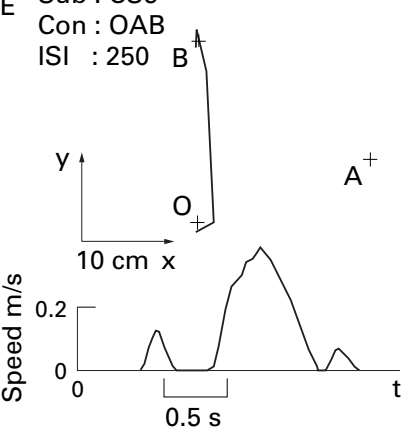

F

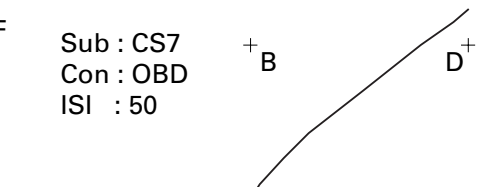

G

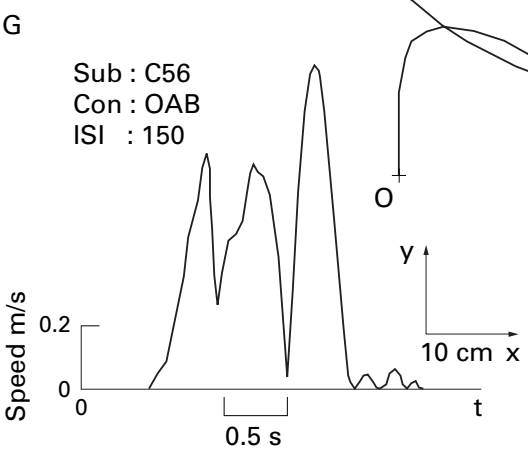

Figure 2 Examples of trajectories performed by patients with Parkinson's disease and control subjects; paths and corresponding tangential velocity profiles are shown. $(A, B)$ Control tasks $O A$ and $O B$ respectively. $(C, D)$ Continuously modified double segment movements (configuration $O A B$ ). The two segments of the trajectory are reflected in two peaks in the velocity profile. (E) A paused modified movement. The subject stopped his movement shortly after initiation $(330 \mathrm{~ms}, 5 \mathrm{~cm})$ and paused for $350 \mathrm{~ms}$. The movement was again initiated in the direction of the final target. $(F)$ Direct movement in configuration $O B D$ (continuously modified movement, fig $1 C$ ). (G) Error in configuration $O A B$. The subject moved as if first responding to the second stimulus, and then moved towards the first target, although he had already reached the vicinity of the final target.
Direct movements to the final target

Direct movements to the final target were common responses to the shift in target location. Figure 2F shows an example of such a movement. Within each group the subjects tended to move more often directly to the final target for shorter ISI values $(0.001<p<0.01$; fig 3B).

Across the subjects, the percentage of direct movements among the switching trials performed by a subject was highly and positively correlated with the RT values of that subject in the control trials (Spearman's correlation analysis: $0.01<p<0.05$ for the patients with Parkinson's disease and for the control subjects, fig 4 for details), suggesting that direct movements reflect a holding strategy (see discussion).

Movement categories, ISIs, and MTs

To obtain a more general picture of the relation between ISI values and the type of movement being generated, we calculated the mean values of ISI for each movement category (referring to the ISI as an independent variable): (a) The direct movements were found in trials in which the mean ISI was 172 (SEM $4 \mathrm{~ms}$ ) for the Parkinson's disease group, and 150 (SEM 5) ms for the control group. (b) The continuously modified double segment movements were found in trials in which the mean ISI was 223 (SEM 4) ms for the Parkinson's disease group, and 212 (SEM $4 \mathrm{~ms}$ for the control group. (c) The paused double segment movements were found in trials in which the mean ISI was 259 (SEM 8) ms for the Parkinson's disease group and 274 (SEM 8) $\mathrm{ms}$ for the control group (see also fig 3).

Within each group the above means were all significantly different from each other (Kruskal-Wallis non-parametric analysis followed by a multiple comparison procedure, $0.001<\mathrm{p}<0.01)$. Across groups only values of mean ISI of the direct movements differed significantly $(0.01<p<0.05)$. Thus, we conclude that if a change in target location occurred roughly within $200 \mathrm{~ms}$ (180 ms for control subjects), the response would be a direct movement, and above roughly $240 \mathrm{~ms}$ there is an increased chance of a paused modified double segment movement.

Following are the values of the modification time (D) obtained in each movement category: (a) In direct movements the mean value of $\mathrm{D}$ was 864 (SEM 22) ms for the Parkinson's disease group, and 877 (SEM 25) ms for the control group. (b) In the continuously modified double segment movements the mean value of D was 275 (SEM 7) ms for the Parkinson's disease group, and 300 (SEM 10) ms for the control group. (c) In the paused double segment movements the mean value of $\mathrm{D}$ was 287 (SEM 18) ms for the Parkinson's disease group, and 299 (SEM 15) ms for the control group. Within each movement category these values were not different across the tested groups ( $p>0.1$, ANOVA). Within each tested group the values of $\mathrm{D}$ in the direct movements were different from the values of $\mathrm{D}$ in the double segment movements $\quad(0.01<\mathrm{p}<0.05$, 

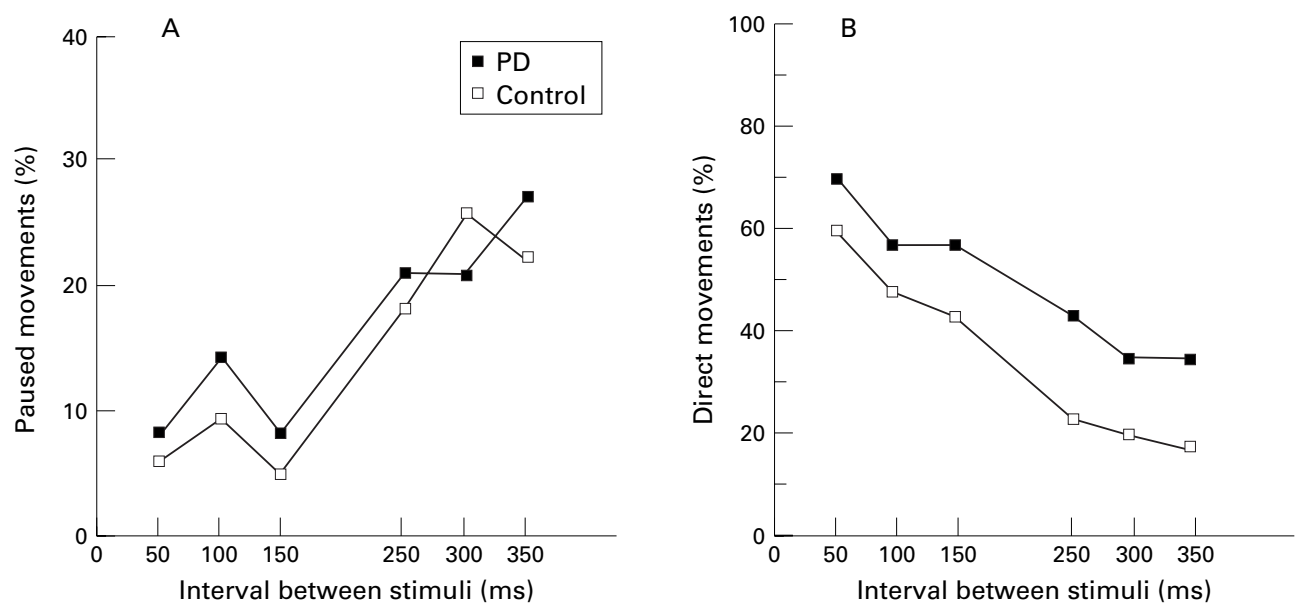

Figure 3 Proportion of paused and direct movements. (A) Paused movements. The graph shows the similarities between the performances of the Parkinson's disease(PD) and control groups. Regression analysis showed a significant correlation between the proportions of paused movements and the values of ISIs $\left(R^{2}=0.82,0.01<p<0.05\right)$ for both groups, and a mutual regression line has the form of: percentage of pauses $=0.6 \times I S I+4.8$. The proportions in the figure are of the total number of double segment movements. (B) Direct movements towards the final target. The proportions of movements directed to the final target of patients with Parkinson's disease and control subjects in switching trials $(n=1437,977$ for Parkinson's disease and control, respectively). Regression analysis: Parkinson's disease regression line: percentage of direct movements $=-0.12 \times I S I+72\left(R^{2}=0.97,0.0001<p<0.001\right)$; control regression line: percentage of direct movements $=-0.14 \times I S I+63\left(R^{2}=0.96,0.0001<p<0.001\right)$.

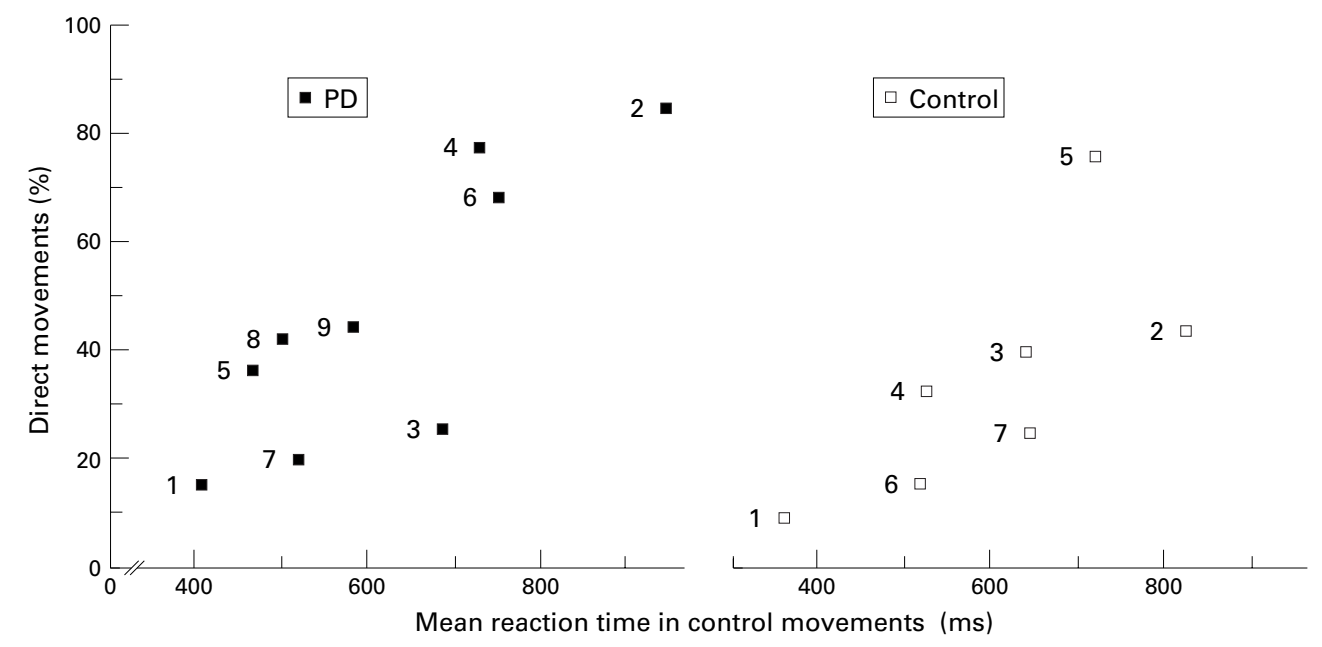

Figure 4 Relation between proportions of direct movements and RT. The proportions of direct movements performed by each subject in relation to the mean RT in control trials. Each square represents one subject (left-Parkinson's disease (PD), right - control), and the number to the left of the squares indicates the index number of the subject. For both groups the proportions of direct movement increased with the increase in the values of $R T$ (Spearman's correlation analysis: $0.01<p<0.05)$.

ANOVA with movement category replacing the group as an effect of the model). The values of $\mathrm{D}$ obtained in the paused double segment movements were similar to those obtained in the continuously modified double segment movements.

Table 2 Values of reaction times (RT) and movement times (MT) (ms)

\begin{tabular}{lll}
\hline Parameter & PD group & Control group \\
\hline RT in control movements & $616(16)$ & $634(13)$ \\
MT in control movements & $1225(18)$ & $1103(16)$ \\
$\mathrm{RT}_{1}$ (switching trials) & $507(8)$ & $522(6)$ \\
$\mathrm{RT}_{2}$ (switching trials) & $926(10)$ & $838(10)$ \\
$\mathrm{RT}_{2} / \mathrm{RT}_{1}$ & $1.93(0.02)$ & $1.67(0.02)^{\star}$ \\
$\mathrm{MT}$ & $1841(32)$ & $1742(40)$ \\
$\mathrm{MT}_{1}$ & $608(8)$ & $505(8)^{\star \star}$ \\
$\mathrm{RT}$ in direct movements & $1032(23)$ & $1027(27)$ \\
\hline
\end{tabular}

${ }^{\star} \mathrm{p}<0.05 ;{ }^{\star \star} \mathrm{p}<0.08$.

tValues (means (SEMs)) are given for configuration OAB. MTs in other switching configurations have comparable values. In all cases, patients with Parkinson's disease (PD) are slightly slower than controls.
QUANTITATIVE ASSESSMENT OF TEMPORAL PARAMETERS

The mean values of the temporal parameters that were investigated are summarised in table 2. In control movements, patients with Parkinson's disease had slightly higher RT and MT values than controls, but the differences were not significant.

\section{Double segment movements}

A MANOVA procedure was performed for all double segment movements on the vector of variables $\mathrm{RT}_{1}, \mathrm{RT}_{2}, \mathrm{RT}_{2} / \mathrm{RT}_{1}$, and MT. No significant difference between the groups was detected, although when each variable was separately inspected (ANOVA), patients with Parkinson's disease had slightly shorter $\mathrm{RT}_{1} \mathrm{~s}$ and longer $\mathrm{RT}_{2} \mathrm{~s}$ and MTs (table 2). For $\mathrm{RT}_{2} \mathrm{~s}$ the ISI effect was highly significant 

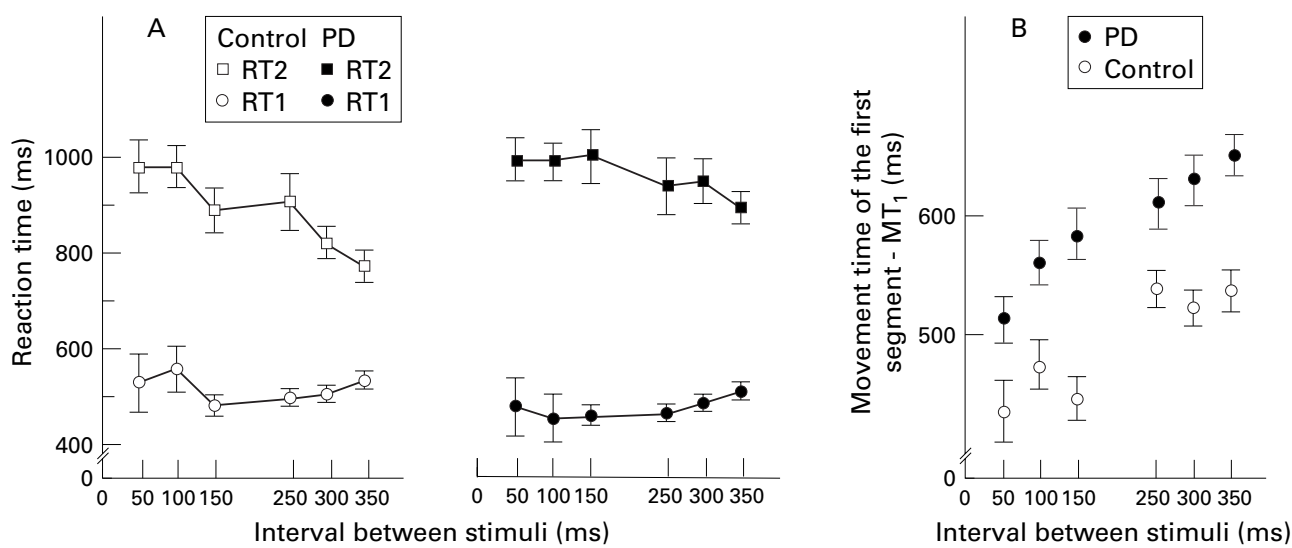

Figure 5 Reaction and movement times as a function of the value of ISI. (A) Mean RT, $s$ and $R T_{2}$ s in double segment movements performed in task configuration OAC. No significant trend in the behaviour of $R T_{1}$ as a function of ISI was found, whereas $R T_{2}$ s decreased significantly as the ISI increased. (B) Mean MTs of the first segment in double segment movements (MT). Patients with Parkinson's disease (PD) were slower than control subjects. Error bars are SEM.

$(0.001<\mathrm{p}<0.01)$ : for higher values of ISI, lower values of $\mathrm{RT}_{2} \mathrm{~s}$ were found (fig $5 \mathrm{~A}$ ). $\mathrm{RT}_{2} \mathrm{~s}$ were significantly prolonged $(\mathrm{p}<0.0001)$ compared with $\mathrm{RT}_{1} \mathrm{~s}$ (paired comparison procedure). The mean $\mathrm{RT}_{2} / \mathrm{RT}_{1}$ ratio for the Parkinson's disease group was 1.93 (SEM 0.02) and for the control group 1.67 (SEM 0.02) $(0.01<\mathrm{p}<0.05)$. In either group, for all subjects the values of this ratio were above 1.0. This is shown in figure 6 , in which $\mathrm{RT}_{2} / \mathrm{RT}_{1}$ ratios are displayed for individual subjects from both subject groups.

We then examined the possibility that the $\mathrm{RT}_{2}$ values that were found in the present study are compatible with a single channel mode of processing (see introduction). This was done by comparing the expected values of $\mathrm{RT}_{2}$ based on the single channel theory to the values actually obtained in the experimental trials. The mean difference between the expected and the measured $\mathrm{RT}_{2}$ was 76 (SEM 13) $\mathrm{ms}$ for the Parkinson's disease group and 126 (SEM 13) $\mathrm{ms}$ for the control group. The mean value of $\mathrm{RT}_{2} / \mathrm{RT}_{2} \exp$ was 0.98 (SEM 0.01) and 0.94 (SEM 0.01) for patients with Parkinson's disease and control subjects, respectively. The difference was not significant. Hence in both groups the measured values of $\mathrm{RT}_{2}$ were in accordance with the single channel theory.

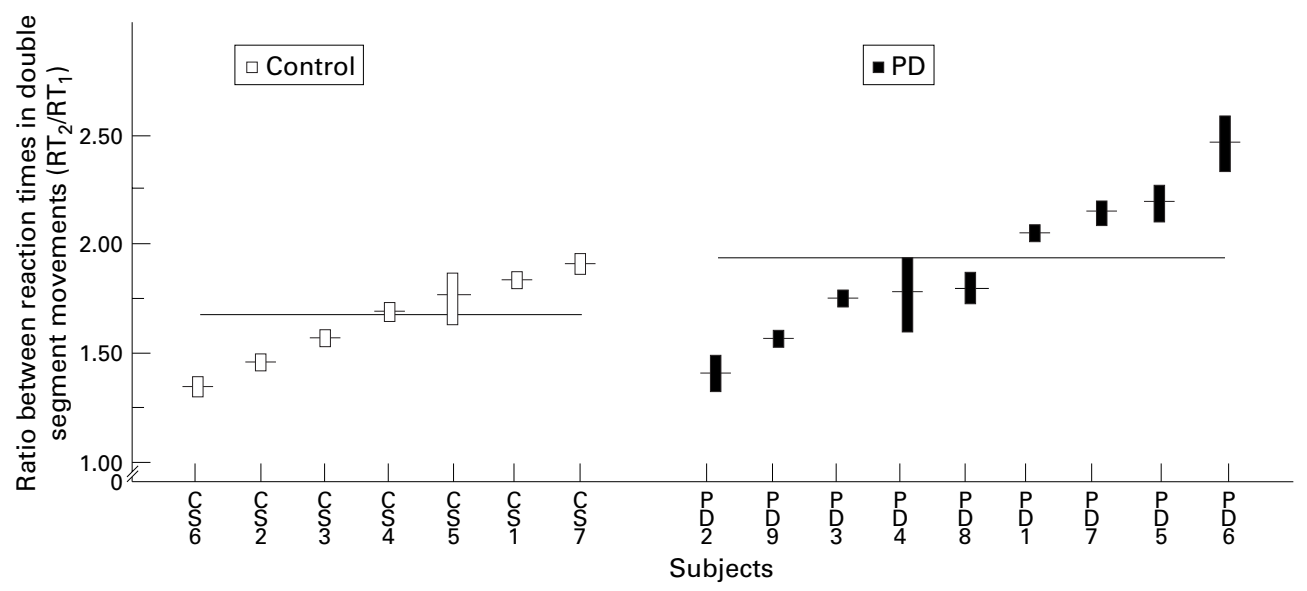

Figure $6 R T_{2} / R T_{1}$ in double segment movements. Means of $R T_{2} / R T_{1}$ ratio for all switching target configurations are presented in an increasing order for the patients with Parkinson's disease (PD) and for the control subjects. Each subject scoring is denoted by a block the length of which represents the SEM. The small bars protruding from the rectangle represent the mean values. The group mean of $R T_{2} / R T_{1}$ for all double segment movements performed by group members is denoted by a horizontal solid line (1.93-Parkinson's disease, 1.67-control $0.01<p<0.05$ ).

Paused double segment movements

In about $16 \%$ of the double segment movements the subjects paused before modifying the direction of movement. The mean value of the pause duration for patients with Parkinson's disease was 244 (SEM 23) ms and for the control group 214 (SEM 14) ms. The difference was not significant.

Movement durations-Overall, the Parkinson's disease group had prolonged MT (table 2) but the difference from the control group was not significant. The mean value of $\mathrm{MT}_{1}$ (the time duration of the first segment in double segment movements) for the Parkinson's disease group was prolonged compared with the control group (this difference was marginally significant, table 2). Both patients with Parkinson's disease and control subjects had longer $\mathrm{MT}_{1} \mathrm{~s}$ for larger ISIs (fig 5B).

\section{Direct movement analysis}

$R T$ in direct movements-These movements usually occurred for short ISIs, and were accompanied by prolonged RTs. The mean values of $\mathrm{RT}$ in the direct movements were similar for both groups (table 2). 


\section{Discussion}

In the present study we have analysed the motor performance of patients with Parkinson's disease and of age matched control subjects in a motor task involving the use of the double step target displacement paradigm. The most important findings in the present study were as follows: (a) In both groups the values of the measured $\mathrm{RT}_{2} / \mathrm{RT}_{1}$ ratios were larger than 1; (b) $\mathrm{RT}_{2} / \mathrm{RT}_{1}$ ratios had larger values in patients with Parkinson's disease than those found in healthy elderly subjects; (c) for both groups measured $\mathrm{RT}_{2}$ values were compatible with those expected according to the single channel theory. Hence, taken together these findings indicate that in both groups there was a relative prolongation of the time period required to prepare a response to the presentation of the second stimulus and this prolongation was more pronounced in Parkinson's disease. Moreover, as discussed below, when comparing the motor performance of Parkinson's disease and elderly subjects to that of young adults in the switching task, ${ }^{89}$ our analysis indicated that patients with Parkinson's disease and elderly subjects have impairments in preparing in parallel motor responses to two consecutively presented visual stimuli, as shown by the existence of a considerable PRP.

Although patients with Parkinson's disease may have prolonged RTs compared with elderly subjects, ${ }^{22}$ we did not find such a phenomenon. Other investigators, particularly in the case of choice reaction times, ${ }^{23-25}$ also reported similarities in RT performance between patients with Parkinson's disease and control subjects. The RTs measured in our study were all choice reaction times. Several earlier studies ${ }^{26}{ }^{27}$ showed prolonged MTs in Parkinson's disease, but in the present study the tendency for bradykinesia was not significant, possibly reflecting the mild to moderate severity of disease in patients participating in the present study.

Our results stress the differences in the performance of the switching tasks of the normal elderly patients and patients with Parkinson's disease compared with young adults. ${ }^{7-10}$ This includes, for example, our findings of the pauses seen in double segment movements, along with the sharp change in movement direction that was found in a substantial number of modified trajectories in the present study. The fact that, on average, the pauses occurred at half the distance between the initial hand position and the first target location and not after reaching the first target, leads us to reject the possibility that those pauses in the modified trajectories occur only after the completion of the first movement and before the initiation of a new movement toward the final target. Moreover, the fact that the ISI, but not the modification time, can serve as a "predictor" for the occurrence of pauses, may point to a mechanism whereby once the first segment of movement of a motor programme reaches an advanced degree of preparation (as in the case of long ISIs), the beginning of its execution is unavoidable, and its modification requires total abortion of movement. Other results (each with non-significant differences between groups), when accumulated, suggest that patients with Parkinson's disease are more impaired when dealing with the switching tasks, as shown, for example, by the relatively greater number of errors or the longer MTs, in particular of the first segment in the switching trials $\left(\mathrm{MT}_{1} \mathrm{~s}\right)$.

The most significant difference between the performance of patients with Parkinson's disease and elderly subjects compared with healthy young adults, was the prolonged values of $\mathrm{RT}_{2} \mathrm{~s}$ compared with $\mathrm{RT}_{1} \mathrm{~s}$, whereas in young adults $\mathrm{RT}_{2} \mathrm{~s}$ were only very slightly longer than $\mathrm{RT}_{1} \mathrm{~s} .{ }^{9}$ Studying the modification of single joint (wrist) movements using a double displacement target paradigm, Montgomery et $a l^{28}$ found, in patients with Parkinson's disease and in elderly subjects, that the RTs to the target shift were shorter than the initial RTs when the shift occurred within $200 \mathrm{~ms}$ after movement onset. The RTs to target shift were comparable with the initial RTs if shifts occurred at the time of the GO signal, or $500 \mathrm{~ms}$ before the GO signal (a signal to move toward a target that had already been presented). The difference between the findings of the present study and those of Montgomery et $a l^{28}$ regarding target switches that occur during the reaction time, is expressed in the fact that we found prolonged RTs to target shift whereas Montgomery et al did not. This difference probably stems from the fact that in their study target locations were known in advance, as the first target, which was illuminated before the GO signal, and the second target (if lit) were always located at fixed locations. Hence, the increased values of $\mathrm{RT}_{2}$ found in our study can be attributed to the existence of a considerable PRP in responding to the second visual stimulus (to the target shift) which may be, among other things, the outcome of the uncertainty in target location.

Direct movements toward the final target were a typical response in the switching trials for both subject groups and were also found in studies of arm trajectory modification in monkeys, ${ }^{6}$ healthy young subjects, ${ }^{79} 14$ elderly subjects, and patients with Parkinson's disease. ${ }^{29}$ Yet the overall proportion of direct movements found in young subjects was much lower than the ones found in the current study in both subject groups. In the present study, direct movements were most likely to occur when the ISIs were shorter than $200 \mathrm{~ms}$ or 180 ms, for patients with Parkinson's disease and control subjects respectively. These figures are comparable with those found by Montgomery et $a l^{29}$ For young adults most of the direct movements were for ISIs $<50-75$ ms. ${ }^{9}{ }^{14}$ What mechanisms are responsible for the phenomenon of directly moving toward the final target? Before discussing several possibilities, we recap the main findings pertinent to this question: $(a)$ direct movements are characterised by prolonged RTs; $(b)$ direct movements appear more often for short ISIs (fig 3); and (c) subjects with long RTs in the control trials perform a higher percentage of direct movements in the switching trials (fig 4). One possibility is that the increased proportion of direct movements 
reflects a holding strategy: If subjects are aware of the possibility that the target may switch to a new location, they may hold their response to the first stimulus and produce a single response to the two stimuli. ${ }^{30}$ It has been shown before in young healthy adults, that in short ISIs, when subjects are aware of the appearance of a second signal, they delay their response to the first stimulus, even when independent bimanual movements are required for each stimulus. ${ }^{31}$

Another mechanism might be related to the dependency of the initial movement direction on the "modification time" (defined as the time interval between the appearance of the second stimulus and the actual response: RT-ISI). It was already suggested that within this period the internal representation of the target location may gradually shift from the first towards the final target location. ${ }^{78}{ }^{10}$ Hence, the longer the modification time, the larger is the distance travelled by the internal target representation and consequently the movement would be initially directed toward the final target. ${ }^{7}$ Given that the reaction times to the second stimulus are relatively prolonged in both patients with Parkinson's disease and elderly subjects, this would explain the high percentage of direct movements associated with short ISIs.

In fig 7 we illustrate the typical dependence of the initial movement direction on the modification time as depicted by the data taken from one patient with Parkinson's disease. It is worth noting, however, that unlike young adults, who tend to generate average trajectories - that is, trajectories that are initially directed along an averaged direction in between the first and final targets whenever the modification time is of an intermediate value ${ }^{32}$-in the present study averaged modified trajectories were hardly found in patients with Parkinson's disease and in elderly subjects (fig 7). This finding, along with the increased percentage of direct movements, may reflect a holding strategy. Further support for this interpretation is found in the positive correlation between RTs in control trials and the high percentage of direct movements. Thus when the subjects did not know a priori whether the forthcoming trial would involve a single or double target displacements, they tended to hold their response to target presentation even in control trials. However, a definite conclusion as to whether a higher percentage of direct movements in parkinsonian and elderly subjects is an outcome of a holding strategy would require further analysis, as some of the above findings are also compatible with the explanation based on a gradual shift of the internal representation of the target location.

In the present study, the increased latency in responding to the target shift was demonstrated, especially in patients with Parkinson's disease, as was reflected in the increased $\mathrm{RT}_{2} / \mathrm{RT}_{1}$. Hence, it seems that a common conclusion from studies using sequential paradigms $^{23} 3334$ and from the present study is that the preparation of a second motor programme is delayed (or at least slowed) in Parkinson's disease, as long as the preparation and/or execution of the first one is still in progress. Apparently, the impairment is either

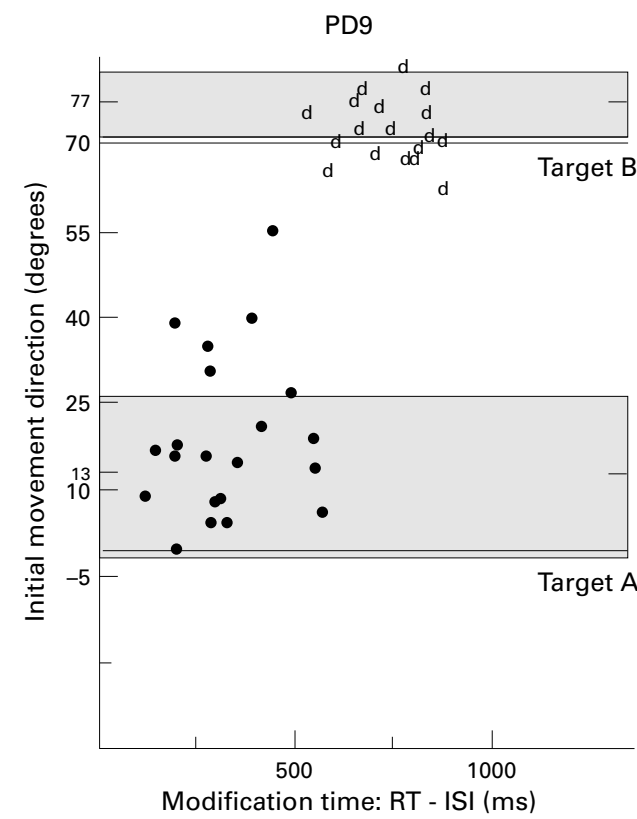

Figure 7 Initial movement direction as a function of modification time; relation between the initial movement direction and the modification time in task configuration $O A B$ for one patient with Parkinson's disease (PD9). The black circles are for double segment movements and the ds are for direct movements (as qualitatively classified). The solid lines (marked by target $A$ and target $B$ ) represent the absolute location of the target. The small bars protruding from the ordinate and the shaded areas denote, respectively, the mean and the SD of the distribution of the initial movement directions as performed in the corresponding control tasks (for details see Henis and Flash ${ }^{10}$ ).

in the perceptual identification of the second stimulus or in the choice and assembly of an appropriate response while still preparing (or executing) the response to the first stimulus. The impairment can also be a combination of these two deficits. The qualitative as well as the quantitative results of the present study show that, unlike young adults, patients with Parkinson's disease and elderly subjects are impaired in their ability to integrate the responses to two successive stimuli. This impairment is more substantial in patients with Parkinson's disease. Another finding which was comparable for both subject groups was an increased tendency to hold the response and to move directly to the final target. The present study has clearly shown the deviation from normal motor switching capabilities shared by both patients with Parkinson's disease and elderly subjects. This is in agreement with an earlier finding by Welford ${ }^{35}$ : "Longer modification times and increased monitoring among older people means that the time between committing themselves to an action and being able to modify it is longer for them than for the younger". Robertson and Flowers ${ }^{36}$ also hypothesised that "Patients may be unable to integrate sequences of action because they cannot be sure what the outcome of any attempted individual movement will be and have to monitor each one as it is executed". Such a "hesitant" approach to movement execution may result in the preference of using a holding strategy, and thus to avoid the need to integrate sequences.

Patients with Parkinson's disease were found to be more affected in their switching perform- 
ance than age matched control subjects. It is possible that as patients with Parkinson's disease have abnormal eye-hand coordination in the sense that a hand movement does not begin until the visual target is foveated, ${ }^{37}$ their capability for trajectory modification is relatively delayed. On the other hand, it was also argued that attention demanding processes are particularly impaired in patients with Parkinson's disease. Thus another possible explanation for the present findings is that when patients with Parkinson's disease are confronted with a dual task performance their movement difficulties are especially exacerbated..$^{25}$ In this context it is worthwhile to mention that according to Marsden, ${ }^{38}$ a general motor plan includes several motor programmes for individual movements as its constituent elements, and involves the smooth integration and sequencing of a series of such elements. If this description is valid, and if the performance in the aging brain deviates from that of the young brain, ${ }^{35}{ }^{39}$ then the results of the present study further suggest a role of the basal ganglia in a smooth and rapid integration of separate motor acts.

Several experimental paradigms have been used and theoretical models proposed for understanding the neuronal mechanism through which the basal ganglia execute this role (for example, Arbib and Dominy ${ }^{40}$ ). Mink, ${ }^{41}$ based on the finding that neurons in the globus pallidus internal segment increase firing before the second and subsequent components of motor sequence, ${ }^{42}$ hypothesised that "for each new component of a sequential movement, new "motor pattern generators" must be turned on and the previous motor pattern generator, as well as other potentially competing motor pattern generators must be inhibited. If the ability to focally select and inhibit competing motor mechanisms is impaired, each component of the movement sequence would be slow and pauses between components might be prolonged". This description strengthens the notion that the results of the present study may reflect physiological impairment in the function of the basal ganglia rather than an adapted control mechansim or compensation strategy.

This research was partially supported by grant No 8800141 from the United States-Israel Binational Science Foundation (BSF), Jerusalem, Israel. This human study was approved in accordance with the ethical standards laid down in the Helsink Declaration. All subjects agreed to participate in this study.

1 Stelmach GE, Worringham CJ, Strand EA. The programming and execution of movement sequences in Parkinson's disease. Intern f Neurosci 1987;36:55-65.

2 Harrington DL, Halland KY. Sequencing in Parkinson's disease. Brain 1991;114:99-115.

3 Benecke R, Rothwell JC, Dick JPR, et al. Disturbance of sequential movements in patients with Parkinson's disease. Brain 1987;110:361-79.

4 Schwab RS, Ghafety ME, Walker S. Control of simultaneous voluntary motor acts in normals and parkinsonism. Arch Neurol 1954;72:591-8.

5 Benecke R, Rothwell JC, Dick JPR, et al. Performances of simultaneous movements in patients with Parkinson's disease. Brain 1986;109:739-57.

6 Georgopoulos AP, Kalaska JF, Massey JT. Spatial trajectories and reaction times of aimed movements: effects of practice, uncertainty and changes in target location. $\mathcal{F} \mathrm{Neu}-$ practice, uncertainty and

7 Van Sonderen JF, Denier Van Der Gon JJ, Gielen CCAM. Conditions determining early modification of motor programs in response to change in target location. Exp Brain Res 1988;71:320-8.
8 Van Sonderen JF, Denier Van Der Gon JJ, Gielen CCAM. Motor programs for goal-directed movements are continuously adjusted according to changes in target location. Exp Brain Res 1989;78:139-46.

9 Flash T, Henis E. Arm trajectory modification during reaching towards visual targets. Fournal of Cognitive Neuroscience 1991;3:220-30.

10 Henis E, Flash T. A computational mechanism to account for averaged modified hand trajectories. In: Moody JE, Hanson SJ, Lippmann RP, eds. Advances in neural information processing systems. San Mateo: Morgan Kaufman, 1992;4:619-26.

11 Prablanc C, Martin O. Automatic control during hand reaching at undetected two dimensional target displacement. F Neurophysiol 1994;67:445-69.

12 Poulton EC. Human manual control. In: Brooks VB, ed. Handbook of physiology, section 1: the nervous system. Vol 2. Motor control. Bethesda, MD: American Physiological SociMotor control. Bethes

13 Soechting JF, Lacquaniti F. Modification of trajectory of a pointing movement in response to a change in target locaion. $\mathcal{F}$ Neurophysiol 1983;49:548-6

14 Gielen CCAM, Heuvel Van Der PJM, Denier Van Der Gon JJ. Modification of muscle activation patterns during fast goal directed arm movements. Fournal of Motor Behavior 1984;16:2-19.

15 Paulignan Y, Mackenzie C, Marteniuk R, et al. Selective perturbation of visual input during prehension movements. 1. The effects of changing object position. Exp Brain Res 1991;83:502-12.

16 Flanagan JR, Ostry DJ, Feldman AG. Control of trajectory modifications in target-directed reaching. Fournal of Motor Behavior 1993;25:140-52.

17 Alexander GE, DeLong MR, Crutcher MD. Do cortical and basal ganglionic motor areas use motor programs to control movement. Behavioral and Brain Sciences 1992;15:644-55.

18 Inzelberg R, Korczyn AD. Hemiballism in Parkinson's Iisease. F Neurol Neurosurg Psychiatry 1995;58:645-8.

19 Hoehn MM, Yahr MD. Parkinsonism: onset, progression and mortality. Neurology 1967;17:427-42.

20 Flash T, Hogan N. The coordination of arm movements: an experimentally confirmed mathematical model. I Neurosci 1985;7:1688-703.

21 Davis R. The limits of the psychological refractory period. $Q$ f Exp Psychol 1956;8:24-38.

22 Evarts EV, Teravainen H, Calne DB. Reaction time in Parkinson's disease. Brain 1981;104:167-86.

23 Bloxham CA, Mindel TA, Frith CD. Initiation and execution of predictable and unpredictable movements in Parkinson's disease. Brain 1984;107:371-84.

24 Sheridan MR, Flowers KA, Harrell J. Programming and execution of movement sequences in Parkinson's disease. Brain 1987;110:1247-71.

25 Goodrich S, Henderson L, Kennard C. On the existence of an attention-demanding process peculiar to simple reaction time: converging evidence from Parkinson's disease. Cognitive Neuropsychology 1989;6:309-31.

26 Hallet M, Khoshbin S. A physiological mechanism of bradykinesia. Brain 1980;103:301-14

27 Flash T, Inzelberg R, Schechtman E, et al. Kinematic analysis of upper limb trajectories in Parkinson's disease. Exp Neurol 1992;118:215-26.

28 Montgomery EB Jr, Nuessen J, Gorman DS. Reaction time and movement velocity abnormalities in Parkinson's disease under different task conditions. Neurology 1991;41: 1476-81.

29 Montgomery EB Jr, Gorman DS, Nuessen J. Motor initiation versus execution in normal and Parkinson's disease subjects. Neurology 1991;41:1469-75.

30 Welford TA. The psychological refractory period and the timing of high speed performance. A review and a theory. Br f Psychol 1952;43:2-19.

31 Way TC, Gottsdanker R. Psychological refractoriness with varying differences between tasks. F Exp Psychol 1968;78: $38-45$.

32 Henis E, Flash T. Mechanism underlying the generation of averaged modified trajectories. Biol Cybern 1995;72:40719.

33 Castiello V, Scarpa M. Perturbation of prehension movement in Parkinson's disease. Mov Disord 1994;9:415-25.

34 Shimizu N, Yoshida M, Nagatsuke Y. Disturbance of two simultaneous motor acts in patients with Parkinsonism and cerebellanataxia. Adv Neurol 1987;45:367-70.

35 Welford TA. Reaction time, speed of performance and age. Ann NY Acad Sci 1988;515:1-17.

36 Roberston C, Flowers KA. Motor set in Parkinson's disease. Roberston C, Flowers KA. Motor set in Parkinson'
f Neurol Neurosurg Psychiatry 1990;53:583-92.

37 Warabi T, Yanagisawa N, Shindo R. Changing in strategy of aiming tasks in Parkinson's disease. Brain 1988;111:497505

38 Marsden CD. The mysterious motor function of the basal ganglia. Neurology 1982;32:514-39.

39 Mortimer JA. Human motor behavior and aging. Ann NY Acad Sci 1988;515:54-65.

40 Arbib MA, Dominey PF. Modeling the roles of basal ganglia in timing and sequencing saccadic eye movements. In: processing in the basal ganglia. Cambridge, Massachusetts: MIT, 1995:149-62.

41 Mink JW. The basal ganglia: focused selection and inhibition of competing motor programs. Prog Neurobiol 1996;50:381 com

42 Brotchie P, Iansek R, Horne MK. Motor function of the monkey globus pallidus. Brain 1991;114:1667-83. 